\title{
Guest host interaction and low energy host structure dynamics in tin clathrates
}

\author{
Sebastian Christensen, ${ }^{1}$ Lasse Bjerg, ${ }^{1}$ Andreas Kaltzoglou, ${ }^{2, a)}$ Fanni Juranyi, ${ }^{3}$ \\ Thomas Fässler, ${ }^{2}$ Tobias Unruh, ${ }^{4, b)}$ and Mogens Christensen ${ }^{1, c)}$ \\ ${ }^{1}$ Center for Materials Crystallography, Department of Chemistry \& iNANO, Aarhus University, \\ Langelandsgade 140, DK-8000 Aarhus C, Denmark \\ ${ }^{2}$ Technische Universität München, Department Chemie, Lichtenbergstraße 4, D-85747 Garching, Germany \\ ${ }^{3}$ Laboratory for Neutron Scattering, Paul Scherrer Institute, CH-5232, Villigen PSI, Switzerland \\ ${ }^{4}$ Technische Universität München, Forschungsneutronenquelle Heinz Maier-Leibnitz FRM, \\ Lichtenbergstr. 1, D-85747 Garching, Germany
}

(Received 23 September 2012; accepted 6 February 2013; published online 25 February 2013)

\begin{abstract}
The two binary clathrates with vacancies ( $\square$ ) $\mathrm{Rb}_{8} \mathrm{Sn}_{44} \square 2$ and $\mathrm{Cs}_{8} \mathrm{Sn}_{44} \square 2$ have been examined using powder inelastic neutron scattering (INS). Rattling energies of Rb and $\mathrm{Cs}$ are found to be similar by both experiment and calculations, $\hbar \omega_{C s} /\left.\hbar \omega_{R b}\right|_{E x p}=0.98(1)$ and $\hbar \omega_{C s} /\left.\hbar \omega_{R b}\right|_{\text {Calc. }}=1.0$, despite the significant mass difference: $m_{C s} / m_{R b}=1.6$, which shows that guest-host interaction is non-negligible for the studied system. For $\mathrm{Rb}_{8} \mathrm{Sn}_{44} \square_{2}$, a low energy phonon mode is observed at $\approx 3.5 \mathrm{meV}$, below the phonon mode which in the literature is attributed to the guest atom. The $3.5 \mathrm{meV}$ mode is interpreted to have significant spectral weight of $\mathrm{Sn}$ host atoms based on temperature dependence and comparison with published theoretical phonon calculations. The record of low thermal conductivity of the tin clathrates can be attributed to the host structure dynamics rather than the guest atom rattling. (C) 2013 American Institute of Physics.
\end{abstract}

[http://dx.doi.org/10.1063/1.4793081]

\section{INTRODUCTION}

A large part of all consumed energy is wasted as heat. Recovering a fraction of this energy would contribute significantly to the reduction of overall energy consumption. Thermoelectrics are ideal for this task, owing to their scalability, reliability, and wide temperature range of application.

Thermoelectric materials are evaluated on the figure of merit, $z T=T S^{2} \sigma / \kappa$, where $T$ is the absolute temperature, $S$ is the Seebeck coefficient, $\sigma$ and $\kappa$ are the electrical and thermal conductivities, respectively. Larger $z T$ corresponds to better energy efficiency, and one way to improve $z T$ is by reducing $\kappa$. Both electrons and phonons in the crystal lattice contribute to the conduction of heat and $\kappa$ is commonly split into two components, $\kappa=\kappa_{e}+\kappa_{l}$.

The use of nanograins to reduce lattice thermal conductivity has resulted in significant improvements in $z T,{ }^{1}$ but the long-term stability of nanoparticles is still a largely unresolved problem. To circumvent this problem, thermoelectric materials with intrinsically low lattice thermal conductivity are of great interest. To a large extent, such materials depend on heavy elements such as $\mathrm{Pb}, \mathrm{Te}$, and $\mathrm{Bi}$ to achieve low $\kappa_{l}{ }^{2,3}$ The extended use of heavy elements poses both environmental and economic problems; hence mechanisms independent of atomic mass and grain boundaries are greatly desired.

\footnotetext{
a) Present address: Department of Chemistry, Heriot-Watt University, Edinburgh EH14 4AS, United Kingdom.

${ }^{b)}$ Present address: University of Erlangen-Nürnberg, Institute of Condensed Matter Physics, D-91058 Erlangen, Germany.

c) Author to whom correspondence should be addressed. Electronic mail: mch@chem.au.dk.
}

Type I clathrates have experienced a surge in interest within recent years due to their promising thermoelectric properties especially caused by a very low thermal conductivity $(\kappa<2 \mathrm{~W} / \mathrm{Km})$. The basic formula for type I clathrates is $A_{8} Y_{\mathrm{x}} M_{46-\mathrm{x}}$, where $M=\mathrm{Si}, \mathrm{Ge}, \mathrm{Sn} ; Y=\mathrm{Al}, \mathrm{Ga}, \mathrm{In}, \mathrm{Ni}, \mathrm{Cu}$, $\mathrm{Zn}, \mathrm{Au}$, vacancy $(\square)$, etc; and A is an alkaline or alkaline earth metal. ${ }^{4-9}$ The $M$ and $Y$ atoms form the host structure consisting of nano cages confining the guest atoms, $A$. The guest atoms have large atomic displacement parameters (ADP) and have, therefore, been termed "rattlers." 10 The guest atoms interact with the phonon dispersion of the host structure, causing decrease in phonon velocity over a range in the low energy regime, which is critical to the conduction of heat. Furthermore, the guest atom will increase the number of high momentum phonon states at low energy. ${ }^{11}$ Phonon states with momentum $\boldsymbol{k}_{1}$ and $\boldsymbol{k}_{2}$ that fulfil $\boldsymbol{k}_{1}+\boldsymbol{k}_{2}>\boldsymbol{K} / 2$, where $\boldsymbol{K}$ is a reciprocal lattice vector, can scatter in umklapp processes. $^{12}$ Thus by decreasing the energy of high momentum states, umklapp processes will become appreciable at lower temperature.

The large primitive unit cell of the clathrates will in itself reduce heat conductance as a result of phonon branches splitting at the zone boundary. ${ }^{13}$

Clathrates of $\mathrm{Si}, \mathrm{Ge}$, and $\mathrm{Sn}$ have significantly lower thermal conductivity than their diamond phase counterparts. ${ }^{14,15}$ It is noteworthy that high temperature lattice thermal conductivity of the diamond phase is very dependent on atomic mass. The clathrate compounds, however, seem largely independent of host structure atomic mass and no clear trend emerges by comparing literature values: $n$-type $\mathrm{Ba}_{8} \mathrm{Ga}_{16} \mathrm{Ge}_{30}: 1.3 \mathrm{~W} / \mathrm{mK}$ at $300 \mathrm{~K},{ }^{16} 1.4 \mathrm{~W} / \mathrm{mK}$ at $250 \mathrm{~K},{ }^{17}$ p-type $\mathrm{Ba}_{8} \mathrm{Ga}_{16} \mathrm{Ge}_{30}: 1.1 \mathrm{~W} / \mathrm{mK}$ at $250 \mathrm{~K},{ }^{17} \mathrm{Ba}_{8} \mathrm{Ga}_{16} \mathrm{Si}_{30}$ : 
$1.1-1.2 \mathrm{~W} / \mathrm{mK}$ at $300 \mathrm{~K},{ }^{18,19} \mathrm{Cs}_{8} \mathrm{Sn}_{44}: 1.0 \mathrm{~W} / \mathrm{mK}$ at $300 \mathrm{~K},{ }^{20}$ $\mathrm{Rb}_{8} \mathrm{Sn}_{44}: 1.1 \mathrm{~W} / \mathrm{mK}$ at $300 \mathrm{~K}^{21}$

$\mathrm{Rb}_{8} \mathrm{Sn}_{44 \square 2}, a=12.0541(1) \AA$ and $\mathrm{Cs}_{8} \mathrm{Sn}_{44 \square 2}, a=12.1063$

(1) $\AA^{21}$ can be described as a type I clathrate structure, which is shown in Figure 1. The framework atoms arrange to form nano cages of two different sizes. The guest atoms situated in the tetrakaidecahedral cage, Figure 1 (blue polyhedra), exhibit the highest ADP and "rattling" which lowers thermal conductivity. $\mathrm{A}_{8} \mathrm{Sn}_{44}$ undergo a reversible order-disorder phase transition at $353 \mathrm{~K} \quad\left(\mathrm{Rb}_{8} \mathrm{Sn}_{44 \square 2}\right)$ and $363 \mathrm{~K} \quad\left(\mathrm{Cs}_{8} \mathrm{Sn}_{44} \square_{2}\right)$. The lowtemperature modification is reported to be a $2 \times 2 \times 2$ superstructure (space group $I a \overline{3} d$ ) of the ideal cubic clathrate with higher ordering of the framework vacancies than in the hightemperature modification (space group $P m \overline{3} n$ ). ${ }^{21,22}$ The host structure vacancies are located on the $6 c$ site, for which the $\mathrm{Sn}$ occupancy is equal to $2 / 3$. Physical property measurements on $\mathrm{Rb}_{8} \mathrm{Sn}_{44 \square 2}$ have shown that the thermal conductivity is not significantly affected by the ordering of vacancies, but the electrical properties are. ${ }^{21}$

Here, we present inelastic neutron scattering (INS) data collected on two partially ordered host structures $\mathrm{Rb}_{8} \mathrm{Sn}_{44} \square_{2}$ and $\mathrm{Cs}_{8} \mathrm{Sn}_{44 \square 2}$. The identical host structures allow for probing the effect of guest atom substitution on the phonon dispersion. Both samples were measured over a range of temperatures to study anharmonic effects and the effect of the order-disorder phase transition.

\section{EXPERIMENTAL DETAILS}

\section{A. Inelastic neutron scattering}

The preparation of the samples used in the presented paper has been described by Kaltzoglou et $a{ }^{21}{ }^{21}$ Samples were characterized with powder X-ray diffraction to confirm phase purity. The INS experiments on powder samples of $A_{8} \mathrm{Sn}_{44 \square 2}$, $A=\mathrm{Cs}$ and $\mathrm{Rb}$ were performed using the instrument TOFTOF at FRM-II, Munich, Germany. ${ }^{23}$ The samples were measured over a range of temperatures: $\mathrm{Cs}_{8} \mathrm{Sn}_{44 \square 2}$ at 298,

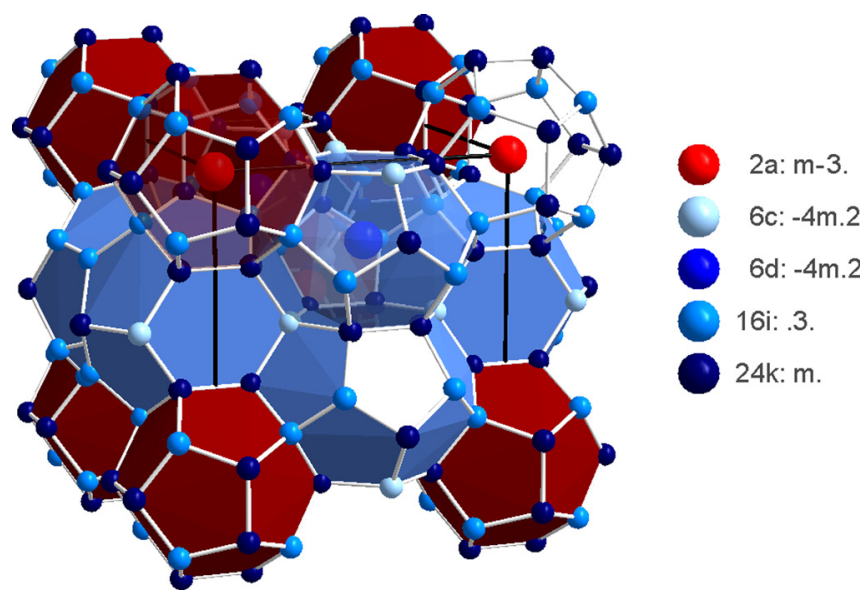

FIG. 1. The average structure of $A_{8} S_{44} \square_{2}, A=R b$, Cs. Polyhedra mark the cages encapsulating the rattling guest atoms on the $6 \mathrm{~d}$ site (tetrakaidecahedra, blue polyhedra) and 2a site (dodecahedra, red polyhedra). The vacancies are located on the $6 \mathrm{c}$ sites (light blue). Ordering of these vacancies break symmetry and form a $2 \times 2 \times 2$ superstructure. The other framework sites are $16 \mathrm{i}$ (blue) and $24 \mathrm{k}$ (dark blue).
323, and $373 \mathrm{~K}$; and $\mathrm{Rb}_{8} \mathrm{Sn}_{44} \square 2$ at $274,298,323$, and $373 \mathrm{~K}$. Extended data collection times were used in the ordered state $(298 \mathrm{~K})$ and above the phase transition (373 K) to achieve high-resolution maps of $(Q, \hbar \omega)$-space and to allow more detailed studies of phonon dispersions. An incident wavelength of $3.5 \AA$ was used in all cases and all shown data are for neutron energy gain (anti-Stokes processes). Data were collected on an empty sample holder and a vanadium standard to account for background and detector efficiency. The collected data were initially treated using the program IDA. ${ }^{24}$

\section{B. Theoretical calculations}

The low energy rattler mode was modelled using density functional theory (DFT) as implemented in VASP. ${ }^{25,26}$ Calculations were performed using the PBE functional ${ }^{27}$ and PAW potentials ${ }^{28,29}$ with a plane wave energy cut off of $315 \mathrm{eV}$ on a $3 \times 3 \times 3 \mathrm{k}$-mesh. The experimental structure was used for calculations (space group $I a \overline{3} d$ ) without relaxing lattice parameters and atomic positions. ${ }^{21}$ Occupancy of the $24 c$ site was reduced from $\sim 0.33$ to 0 to form the hypothetical $A_{8} \mathrm{Sn}_{43} \square_{3}, A=\mathrm{Cs}, \mathrm{Rb}$ phase which is disorder free.

\section{RESULTS AND DISCUSSION}

The neutron-weighted generalized phonon density of states (GDOS) has been calculated from the measured scattering function $S(\hbar \omega, Q)$, Figure 2, within the one-phonon, incoherent approximation,

$$
G(\hbar \omega)=\frac{C}{\Delta Q} \int_{Q_{\min }(\hbar \omega)}^{Q_{\max }(\hbar \omega)} \frac{\omega}{Q^{2}} S(\hbar \omega, Q, T) \times n(\hbar \omega, T)^{-1} d Q .
$$

$n(\hbar \omega, T)$ is the Bose-Einstein (BE) factor which accounts for thermal population of the phonon states at energy $\hbar \omega$ and temperature T. $S(\hbar \omega, Q, T)$ is integrated from the smallest measured momentum transfer $Q_{\min }$ to the maximal $Q_{\max }$

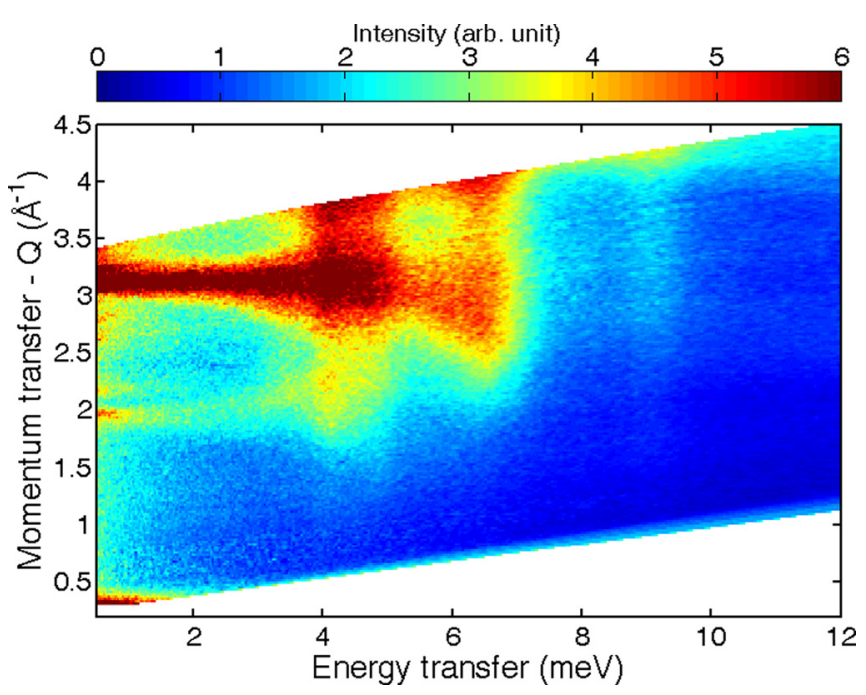

FIG. 2. Scattering function $S(\hbar \omega, Q, T)$ for $\mathrm{Rb}_{8} \mathrm{Sn}_{44} \square_{2}$ at $373 \mathrm{~K}$ showing parts of momentum-energy space accessible to the instrument. Colour bar indicates the relative intensity of scattering on a linear scale. 
and then normalized to the integration area $\Delta Q=$ $Q_{\max }-Q_{\min }$. C is a scale factor which has not been determined for the reported data. ${ }^{30,31}$ Although the measured Qspace samples more than 30 symmetry in-equivalent Brillouin zones, the incoherent approximation is not well fulfilled as the main contribution to the dynamical scattering function comes from only a few Brillouin zones: (530), (321), (532), and (431), indexed according to high temperature structure. However, the strict fulfilment of the incoherent approximation is only important when comparing GDOS directly to calculations.

The GDOS for both compounds shows a low energy feature below $5 \mathrm{meV}$, Figures 3(a) and 3(c), which is typical for the clathrates described in literature. ${ }^{6,7,32}$ The literature assignment of modes is used, i.e., the peak at $4-5 \mathrm{meV}$ is dominated by contributions from the rattling guest atoms and it is referred to as the "guest mode," though also host structure atoms will be contributing. High energy peaks $(>6 \mathrm{meV})$ are predominantly attributed to host structure vibrations. ${ }^{11,33,34}$ Peak positions are determined from the dynamic structure factor $S(\hbar \omega, T=298 \mathrm{~K})$ obtained by averaging $S(\hbar \omega, Q, T)$ over the accessible momentum-space, Figures 3(b) and 3(d). The temperature dependence of dynamic structure factor of all experiments has been rescaled to $\mathrm{T}=298 \mathrm{~K}$ to ensure comparability between datasets obtained at different temperatures, $S(\hbar \omega, T=298 \mathrm{~K})$ $=S(\hbar \omega, T) \cdot n(\hbar \omega, 298 K) / n(\hbar \omega, T), \quad$ where $\quad n(\hbar \omega, T)$ $=\left(\exp \left(\frac{\hbar \omega}{k_{B} T}\right)-1\right)^{-1}$ is the BE-factor. We choose to fit the $S(\hbar \omega)$ and not the GDOS because features at low energies are thereby enhanced. Each region has been fitted using 1-3 Gaussians with a linear contribution as indicated in Figures 3(b) and 3(d). The number of Gaussians was chosen to best reproduce the peak shape. Each peak is the projection of multiple 3D phonon branches into 1D, so no direct physical meaning can be extracted from the number of Gaussians; the aim is to get reliable peak profile to extract accurate peak positions. The extracted peak position is the global maximum of the fitted function. Results are presented in Figure 4.

The dynamic structure factor shows no visible change upon crossing the structural phase transition around $360 \mathrm{~K}$.
The guest mode, Figures 3(b) and 3(d), is significantly broader than the instrumental resolution and appears wider than observed for other clathrates. ${ }^{32,36}$ Instrument resolution of the elastic line is $0.24 \mathrm{meV}$ (measured). The energy resolution $(\Delta E)$ at different non-zero energy transfers has been calculated based on Unruh et al.: ${ }^{23} \Delta E(4 \mathrm{meV})=0.37 \mathrm{meV}$ and $\Delta E(10 \mathrm{meV})=0.63 \mathrm{meV}$. The width of all fitted peaks exceeds the instrumental resolution. The broad experimental peak shape indicates contributions from several or highly dispersive modes in addition to the contribution from finite phonon lifetimes and instrumental effects.

The temperature dependence of phonon modes gives information about the anharmonicity of the atomic potentials. As a result of thermal expansion phonon energies are generally expected to decrease (soften) in response to heating. ${ }^{37}$ Such behaviour is observed for the host structure peak at $\approx 6.4 \mathrm{meV}$ and $9 \mathrm{meV}$ Figures 4 (b) and 4(c). In contrast, the phonon energy of the guest mode peak at $\approx 4.5 \mathrm{meV}$ increases in response to heating (hardens), Figure 4(a). Hardening is a characteristic property of phonon modes which have large guest atom contributions and is observed in several guest-host systems. ${ }^{7,38,39}$ The unusual temperature dependence is indicative of a U-shaped potential of an atom confined inside an oversized nano cage ${ }^{40}$ which is an intermediate between a harmonic potential with no $\mathrm{T}$ dependence and an infinite well potential. In the present study, we intend to use the temperature dependence of phonon modes as an experimental indicator to whether guest or host atoms are the dominant contributor to phonon modes in a guest-host system. The degree of anharmonicity of the guest atom potentials is estimated from the slope of linear fits (Figure 4(a): $\mathrm{Rb}_{8} \mathrm{Sn}_{44} \square_{2}$ : $16(6) \cdot 10^{-4} \mathrm{meV} / \mathrm{K}, \mathrm{Cs}_{8} \mathrm{Sn}_{44} \square_{2}: 12(1) \cdot 10^{-4} \mathrm{meV} / \mathrm{K}$. Both slopes are larger than obtained for $\mathrm{n} / \mathrm{p}-\mathrm{Ba}_{8} \mathrm{Ga}_{16} \mathrm{Ge}_{30}$, but half the value obtained for $\mathrm{Sr}_{8} \mathrm{Ga}_{16} \mathrm{Ge}_{30}{ }^{7}$. For the host structure peaks, see Figure 4(b), the anharmonic effect is smaller and the slopes are $\mathrm{Rb}_{8} \mathrm{Sn}_{44} \square_{2}:-10(2) \cdot 10^{-4} \mathrm{meV} / \mathrm{K}, \mathrm{Cs}_{8} \mathrm{Sn}_{44} \square_{2}$ : $-4(1) \cdot 10^{-4} \mathrm{meV} / \mathrm{K}$. The anharmonic terms in the atomic potentials are important for the thermal conductivity as they facilitate phonon-phonon scattering; ${ }^{12}$ however, no clear connection has been made between anharmonicity of the guest mode and thermal conductivity of the metal clathrates. $^{7}$

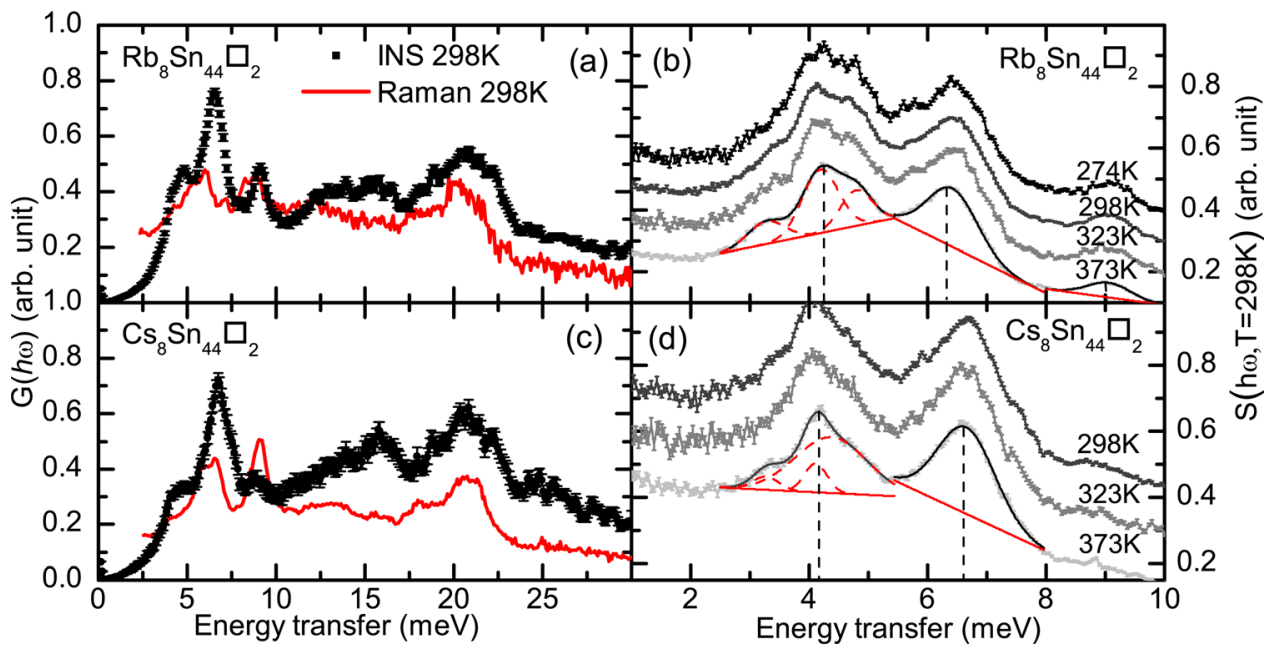

FIG. 3. Generalized phonon densities of states (GDOS) for $\mathrm{Rb}_{8} \mathrm{Sn}_{44} \square_{2}$ (a) and $\mathrm{Cs}_{8} \mathrm{Sn}_{44} \square 2$ (c) at $298 \mathrm{~K}$ plotted together with Raman data corrected for BoseEinstein statistics. Raman data from Shimizu et $a l^{35}$ Dynamic structure factor $S(\hbar \omega)$ of $\mathrm{Rb}_{8} \mathrm{Sn}_{44} \square_{2}$ (b) and $\mathrm{Cs}_{8} \mathrm{Sn}_{44} \square_{2}$ (d) rescaled to $298 \mathrm{~K}$ for comparison. Full black lines are fits to data. Individual Gaussians are shown as dashed red lines. Dashed black lines indicate the extracted peak maxima. 


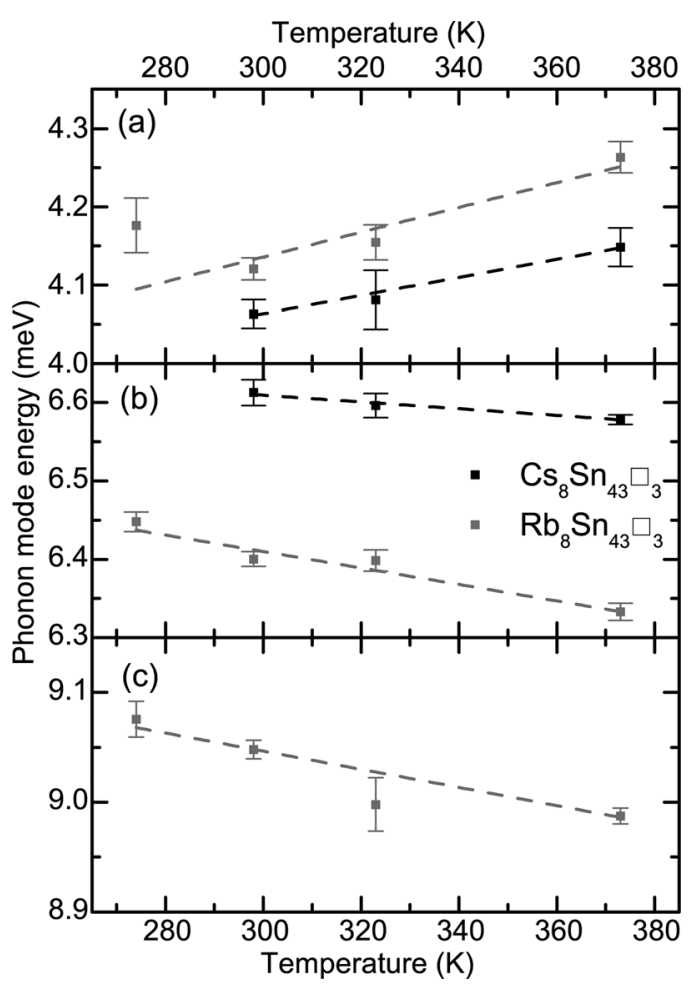

FIG. 4. Peak positions of the dominant peaks in Figure 3 related to the guest atom (a) and host structure (bc). $\mathrm{Rb}_{8} \mathrm{Sn}_{44} \square_{2}$ (grey) and $\mathrm{Cs}_{8} \mathrm{Sn}_{44} \square_{2}$ (black). $9 \mathrm{meV}$ peak (c) was only clearly resolved for $\mathrm{Rb}_{8} \mathrm{Sn}_{44} \square_{2}$. Dashed lines are linear fits to the data points.

The average ratio of experimentally determined guest atom energies is $\hbar \omega_{C S} /\left.\hbar \omega_{R b}\right|_{I N S}=0.98(1)$, despite the large mass difference: $m_{C s} / m_{R b}=1.6$. In the harmonic approximation, the energy of the phonon mode is related to the mass by $\hbar \omega \propto \sqrt{K / m}$, where $K$ is the spring constant and $m$ is the atomic mass. ${ }^{12}$ Assuming identical spring constants, $K$, the guest atom energy ratio becomes $\hbar \omega_{C s} /\left.\hbar \omega_{R b}\right|_{\text {Harm. }}=0.79$ which clearly shows that such an assumption is invalid. Substitution of the guest atom will affect the guest atom to cage volume fraction, $V_{\text {guest }} / V_{\text {cage }}$. Reducing the cage volume by physical or chemical pressure has been demonstrated to increase energy of the guest mode. ${ }^{7}$

A simple model was used to investigate the effect of the volume fraction on $K$. DFT calculations were performed on the ordered $\mathrm{A}_{8} \mathrm{Sn}_{43} \square 3$ phase (space group $I a \overline{3} d$ ), where the $6 d$-site $A$ atoms were moved while preserving symmetry. The experimental structures from Kaltzoglou et al. ${ }^{21}$ were used. Specifically, positions were set to $(a / 8, a / 4-d, d)$ for varying $d$, where $a$ is the unit cell lattice parameter. We chose this set of displacements because larger ADPs were found for the $\mathrm{y}$ and $\mathrm{z}$ directions. Calculations showed a welldefined minimum close to the experimental guest atom position, demonstrating that a stable guest atom position was obtained. A second order polynomial of the form $E=1 / 2 \times K\left(\sqrt{2} \cdot\left(d-d_{0}\right)\right)^{2}+E_{0}$ was fitted to the obtained energies, $E$. The factor of $\sqrt{2}$ comes from the fact that both the $y$ and $z$ coordinates are changed by $d$. As can be seen in Figure 5, good fits with second order polynomials

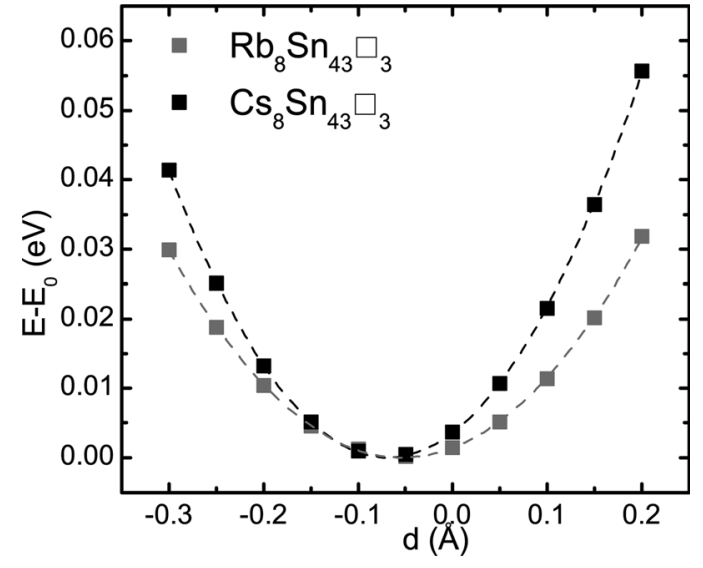

FIG. 5. Potential energy of the rattling guest atoms in $\mathrm{Rb}_{8} \mathrm{Sn}_{43} \square_{3}$ (grey) and $\mathrm{Cs}_{8} \mathrm{Sn}_{43} \square_{3}$ (black) for the points $(\mathrm{a} / 8, \mathrm{a} / 4-\mathrm{d}, \mathrm{d})$. Dashed lines are $2^{\text {nd }}$ order polynomial fits to the calculated values.

were obtained. A spring constant ratio of $K_{C s} / K_{R b}=1.57$ was found, giving a ratio between the vibrational energies of $\hbar \omega_{C s} /\left.\hbar \omega_{R b}\right|_{\text {Calc. }}=1.00$, close to the observed value. The actual calculated vibrational energies were found to be around $4.9 \mathrm{meV}$. The higher values compared to the experimental findings can be attributed to the simplicity of the model.

Making the assumption that $K \propto\left(V_{\text {guest }} / V_{\text {cage }}\right)$ gives an expected spring constant ratio of $K_{C S} / K_{R b}=1.47$. The guest atom volume $V_{\text {guest }}$ was calculated from the Pauling ionic radii of $\mathrm{Cs}^{+}(169 \mathrm{pm})$ and $\mathrm{Rb}^{+}(148 \mathrm{pm}){ }^{41}$ This spring constant ratio is comparable to the value obtained using DFT calculations.

Substituting $\mathrm{Cs}$ for $\mathrm{Rb}$ has a larger effect on the energy of host structure vibrations compared to the guest atom vibrations. The host mode energy for $\mathrm{Cs}_{8} \mathrm{Sn}_{44} \square_{2}$ is on average increased by 0.22 (3) meV compared to $\mathrm{Rb}_{8} \mathrm{Sn}_{44} \square_{2}$. The larger size of Cs acts to stiffen the host structure, causing an overall energy increase of host structure vibrational energies.

The scattering function, $S(\hbar \omega, Q)$ of $\mathrm{Rb}_{8} \mathrm{Sn}_{44} \square_{2}$, Figure 2 , exhibits a faint flat mode around $\approx 3.5 \mathrm{meV}$ which is below the energy assigned to the rattling guest atom, $>4 \mathrm{meV}$. To verify the presence of a low energy phonon mode below the guest rattling mode, the scattering function was integrated in steps of $0.3 \AA^{-1}$ for both compounds, Figure 6. For $\mathrm{Rb}_{8} \mathrm{Sn}_{44} \square_{2}$ at $373 \mathrm{~K}$ a low energy peak is clearly separated from the "guest" peak over a large range $\left(Q=2.2-3.4 \AA^{-1}\right)$, but at $298 \mathrm{~K}$ the two peaks appear to merge. For $\mathrm{Cs}_{8} \mathrm{Sn}_{44} \square_{2}$ a peak at $3.5 \mathrm{meV}$ can only be separated at $Q=3.1 \AA^{-1}$. Counting statistics is significantly lower for $\mathrm{Cs}_{8} \mathrm{Sn}_{44} \square_{2}$ due to smaller sample amount compared to $\mathrm{Rb}_{8} \mathrm{Sn}_{44} \square_{2}$. The inferior data quality makes fewer details visible. The background signal from the empty sample holder and cryostat has been integrated and plotted in Figures 6(e) and 6(f). There are no strong features in the background below $10 \mathrm{meV}$, therefore the peak at $\sim 3.5 \mathrm{meV}$ originates from sample.

Integrating the scattering function over the interval: $Q=2.3-2.55 \AA^{-1}$ corresponding to the Brillouin zone boundary between the strongest reflections gave the maximal 


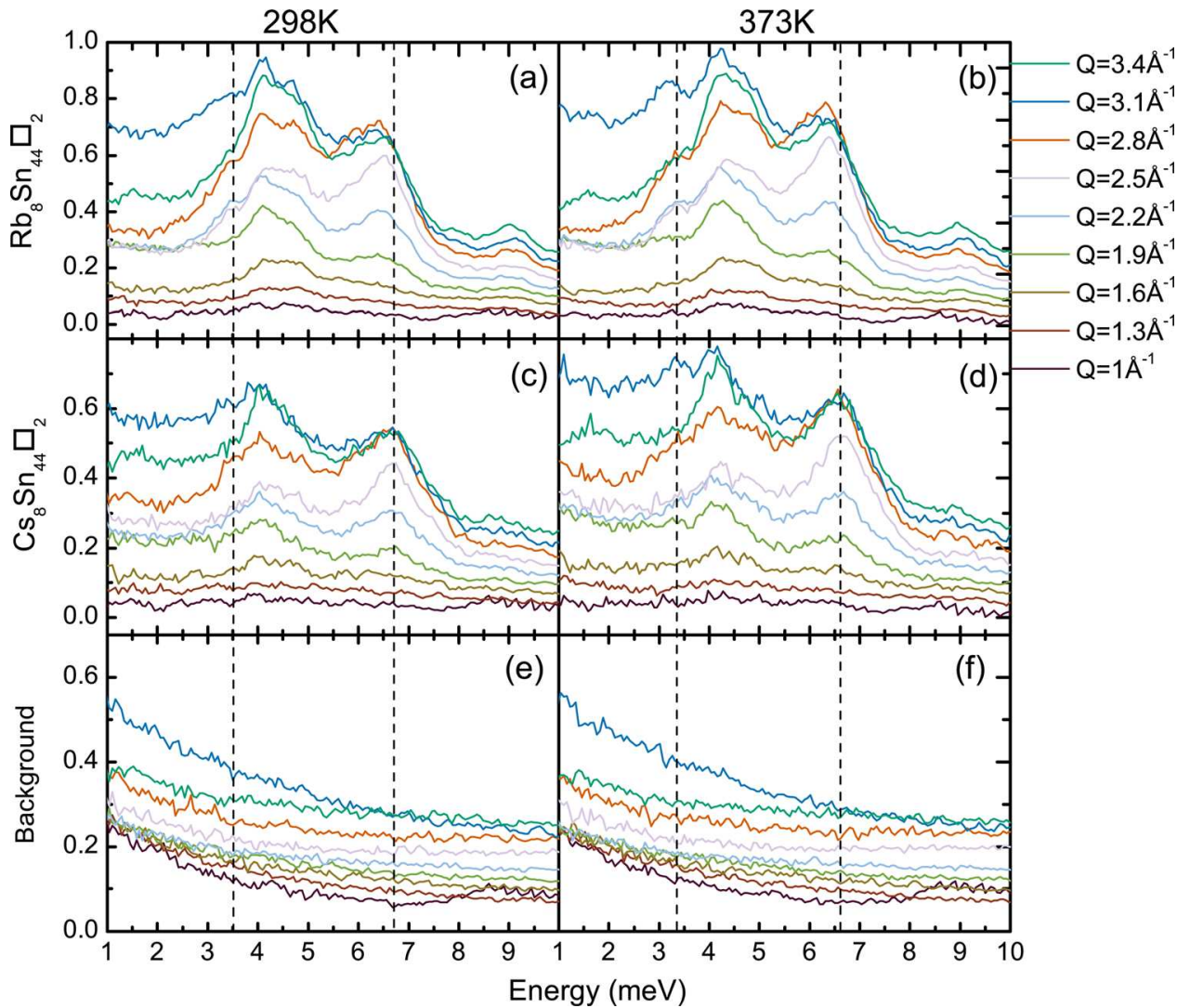

FIG. 6. Scattering function corrected for background integrated over intervals of $0.3 \AA^{-1}$ centred on momentums, Q, given in the legend. (ab) $\mathrm{Rb}_{8} \mathrm{Sn}_{44} \square_{2}$, (cd) $\mathrm{Cs}_{8} \mathrm{Sn}_{44} \square 2$, and (ef) empty sample container.

contrast between neighbouring phonon modes. For $\mathrm{Rb}_{8} \mathrm{Sn}_{44} \square 2$ the low energy mode at $\approx 3.5 \mathrm{meV}$ is resolved both below $(298 \mathrm{~K})$ and above $(373 \mathrm{~K})$ the structural phase transition, Figure 7. In $\mathrm{Cs}_{8} \mathrm{Sn}_{44} \square 2$ no low energy phonon mode can be separated from the guest mode. Fitted peak positions for $\mathrm{Rb}_{8} \mathrm{Sn}_{44} \square_{2}$ data are given in Figure 8. It is striking that the peak at $\approx 3.5 \mathrm{meV}$ softens upon heating like the host structure-peak $(6.5 \mathrm{meV})$, while in contrast to the hardening guest peak $(4 \mathrm{meV})$. The similar temperature dependence of the peaks at $3.5 \mathrm{meV}$ and $6.5 \mathrm{meV}$ is interpreted as a testament to their common origin, i.e., the peak at $3.5 \mathrm{meV}$ has dominant spectral weight of host structure atoms. Similar low-energy host modes are usually not observed theoretically nor experimentally for germanium or silicon type I clathrates. ${ }^{6,7,11,32-34,42}$ In a single study of $\mathrm{Ba}_{8} \mathrm{Zn}_{8} \mathrm{Ge}_{38}$, a weak shoulder to the guest mode has been observed toward lower energy; however, the authors do not comment on its origin. ${ }^{36}$

The possible effect of a structural transition must be addressed as data are compared above and below a structural phase transition. The primitive unit cell of the low temperature phase $(I a \overline{3} d)$ is expanded 4-fold compared to the high temperature phase $(P m \overline{3} n)$. Consequently the (primitive) Brillouin zone volume is reduced, causing a back-folding and possibly splitting of phonon branches. The phonon dispersion of the $A_{8} S_{44} \square 2$ low temperature phase is expected

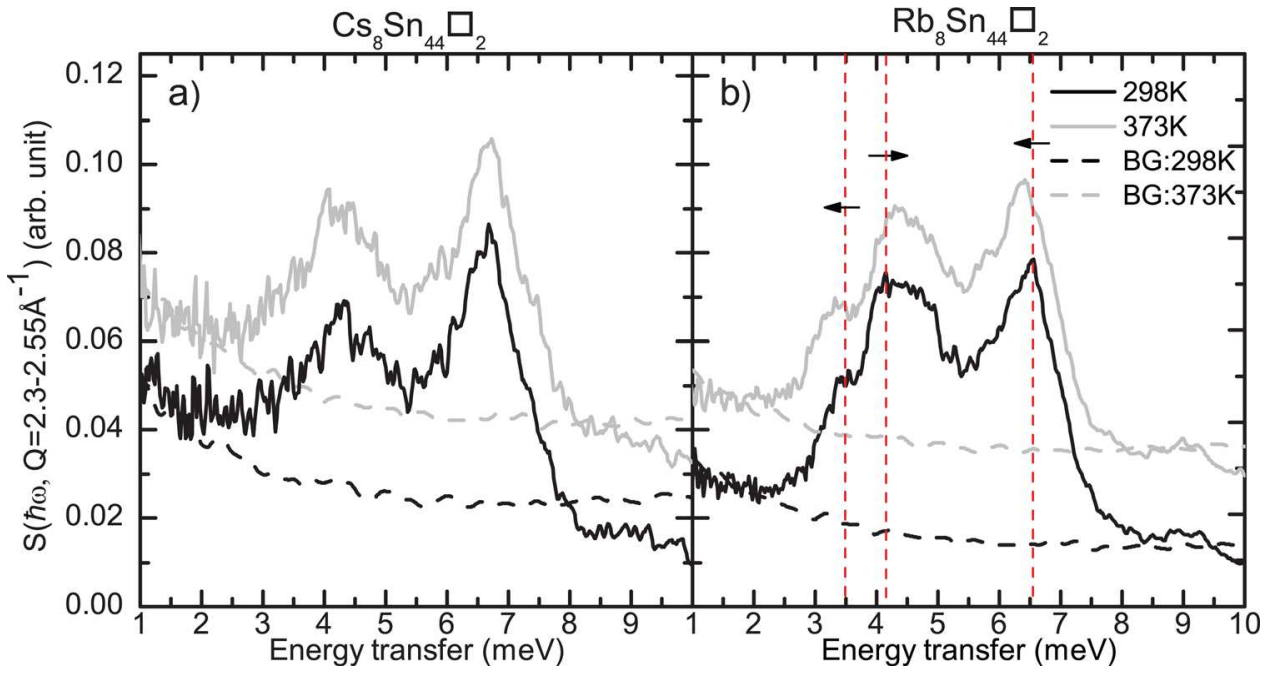

FIG. 7. (Full lines) Integration of the background subtracted scattering function from $Q=2.3 \AA^{-1}$ to $Q=2.55 \AA^{-1}$ for $\mathrm{Cs}_{8} \mathrm{Sn}_{44} \square_{2}$ (a) and $\mathrm{Rb}_{8} \mathrm{Sn}_{44} \square 2$ (b). $298 \mathrm{~K}$ (black) and $373 \mathrm{~K}$ (grey). A peak at $\approx 3.5 \mathrm{meV}$ is clearly seen for $\mathrm{Rb}_{8} \mathrm{Sn}_{44} \square$. The data have been rescaled to $298 \mathrm{~K}$. Positions of the peaks at $3.5 \mathrm{meV}, 4 \mathrm{meV}$, and $6.5 \mathrm{meV}$ have been fitted and plotted in Figure 8. Arrows show the movement of peaks upon heating. (Dashed lines) Background signal from empty sample container and sample environment. 


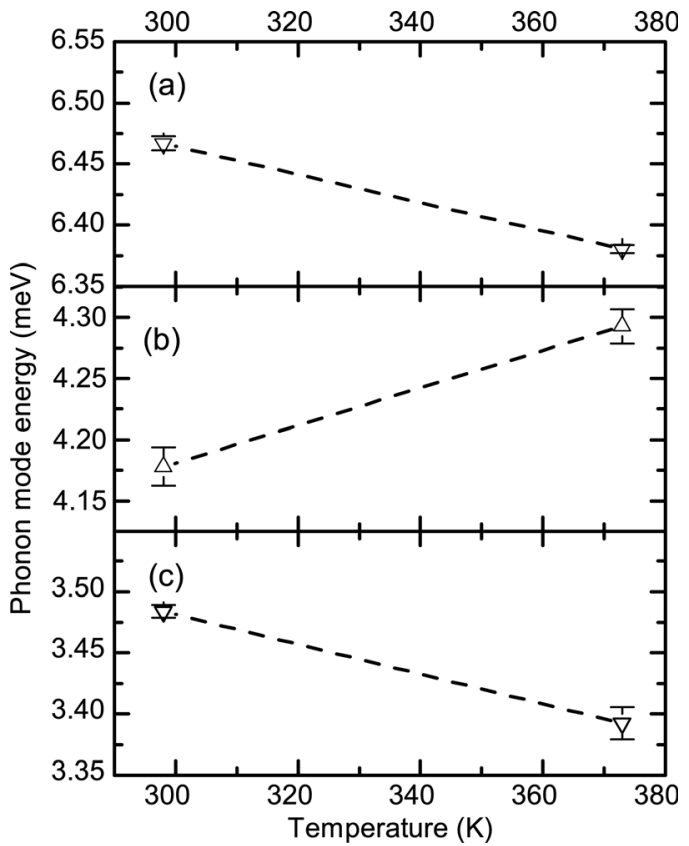

FIG. 8. Peak positions from Figure 7(b) obtained by fitting $S(\hbar \omega$, $Q=2.3-2.55 \AA^{-1}$ ) with Gaussians and a linear background. Peaks at $6.5 \mathrm{meV}$ (a) and $3.5 \mathrm{meV}$ (c) both move toward lower energy at higher temperature in contrast to the "guest peak" at $4 \mathrm{meV}$ (b), which moves to higher energy. Dashed lines are guides to the eye.

to be comparable to the isostructural $\mathrm{Ba}_{8} \mathrm{Ge}_{43} \square 3$ for which the dispersion has been calculated. ${ }^{31}$ The calculations do indeed show splitting of low energy phonon branches in the $[0,0,0] \rightarrow[0.5,0,0]$ direction; however, the splitting is too localized in reciprocal space to produce an observable van Hove singularity in the calculated GDOS. For this reason, we do not expect the structural phase transition to have a visible effect on the measured scattering function. If the structural transition had a visible effect it should be manifested as a peak in the low energy region, $\mathrm{E}<4 \mathrm{meV}$; however, the peak should only be present below the phase transition, but absent above it. In the present case the peak below $3.5 \mathrm{meV}$ is observed both below $(298 \mathrm{~K})$ and above the phase transition $(373 \mathrm{~K})$. This is a strong indication that the structural transition has no effect on the observations.

Theoretical calculations have been published for the hypothetical compound $\mathrm{Cs}_{8} \mathrm{Sn}_{46} \cdot{ }^{34}$ The calculations were carried out in space group $P m \overline{3} n$ and did not include the symmetry reducing ordering of vacancies. The calculations show transverse acoustic phonons to reach the zone boundary without interacting with the guest mode. In other words, host structure atoms have significant contributions to the phonon density of states at low energy; both below and directly above the guest atom mode. These theoretical results support our experimental findings of a low energy phonon mode with lattice like temperature dependence, below the guest mode. The experimentally observed peak at $3.5 \mathrm{meV}$ is interpreted to be the transverse acoustic branch flattening at the zone boundary. In general, the calculated energies are lower than the experimental values. According to calculations, the low energy mode is expected to be present in both $\mathrm{Rb}_{8} \mathrm{Sn}_{44} \square 2$ and $\mathrm{Cs}_{8} \mathrm{Sn}_{44} \square 2$; however, only for $\mathrm{Rb}_{8} \mathrm{Sn}_{44} \square 2$ are we able to resolve this mode. The most probable cause is the inferior quality of the $\mathrm{Cs}_{8} \mathrm{Sn}_{44} \square 2$ data. Based on the assumption that the transverse acoustic branch reaches the zone boundary at $3.5 \mathrm{meV}$ and adopts a sinusoidal dispersion we estimate a maximal transverse phonon speed of $3.2 \cdot 10^{3} \mathrm{~m} / \mathrm{s}$ in the [100]-direction.

The phonon dispersion of the skutterudite $\mathrm{CeOs}_{4} \mathrm{Sb}_{12}$ has been studied using triple axis neutron scattering. ${ }^{43}$ The measured phonon dispersion shows no signs of a rattling mode interacting with the acoustical branches. This can be interpreted as the acoustic branches not to reaching the guest mode energy owing to heavy framework atoms. ${ }^{13}$

Phonon-dispersions of the type II clathrates $A_{8} \mathrm{Ga}_{8} \mathrm{Si}_{128}$, $A=\mathrm{Cs}$, Rb have been calculated by ab initio methods. ${ }^{42}$ The type I and II clathrates are closely related in the sense that both contain loosely bonded guest atoms in similarly sized cages. Despite the higher mass of $\mathrm{Cs}$, these calculations show Cs to have the highest rattler energy. The authors explain this by increased interaction between Cs and the host structure caused by the larger guest atom size. These calculations corroborate the trends of our experimental and theoretical calculations; however, any direct comparison is not possible due to the structural differences. Though Myles et $a l{ }^{42}$ do not comment on it, the energies of host modes in $\mathrm{Cs}_{8} \mathrm{Ga}_{8} \mathrm{Si}_{128}$ are slightly increased, compared to $\mathrm{Rb}_{8} \mathrm{Ga}_{8} \mathrm{Si}_{128}$. Although this effect is small and only visible at high energy, it is in good agreement with our findings, Figure 4(b).

Both $\mathrm{Rb}_{8} \mathrm{Sn}_{44} \square 2$ and $\mathrm{Cs}_{8} \mathrm{Sn}_{44} \square 2$ have been investigated using Raman scattering. ${ }^{35}$ Low energy modes are observed in the energy range $3-5 \mathrm{meV}$. Raman scattering observes only phonon modes at the Brillouin zone centre which fulfil specific selection rules. Comparison with Raman data would be ideal to establish whether the low energy modes in the present case are originating from the zone centre or zone boundary. Observation at the zone centre would point to optic modes with guest atom character, whereas no Raman signal would be observed if the signal was originating from the acoustic phonons going flat at the zone boundary. However, the data quality of the Raman data is insufficient at low energy to resolve the modes clearly and make decisive conclusions. The most distinct peaks in the Raman spectrum are observed at 6.6, 9, and $20 \mathrm{meV}$, which is in agreement with our data, Figures 3(a) and 3(c).

The type I clathrate $\mathrm{Cs}_{8} \mathrm{Ga}_{8} \mathrm{Sn}_{38}$ has been investigated by Raman scattering and theoretical phonon calculations. ${ }^{44}$ Authors conclude that the low energy Raman active modes below $5 \mathrm{meV}$ are caused by $\mathrm{Cs}$ rattling. However, their calculations for guest free $\mathrm{Sn}_{46}$ show transverse acoustic phonon branches to reach the zone boundary in the range $2.5-3.75 \mathrm{meV}$ which is below the guest rattling modes. ${ }^{44}$

Based on multi-temperature INS measurements and comparison to theoretical calculations of Tse et al., ${ }^{34}$ we interpret the peak at $3.5 \mathrm{meV}$ to be the transverse acoustic branch reaching the Brillouin zone border below the guest mode energy. According to the "avoided crossing" model lowering of the room temperature thermal conductivity of clathrates happens through hybridization of the guest 
rattling modes with the host structure acoustic phonon branches. $^{11}$ In the case of $\mathrm{Sn}$ clathrates, the transverse acoustic branch is merely indirectly affected by the presence of guest atoms, owing to its intrinsically low energy. Only the longitudinal branch has sufficiently high energy to possibly hybridize with the guest mode. Due to the lack of interaction, the guest atoms will have less effect in the reduction of the phonon velocity. The guest atom will create high momentum phonon states (with low group velocity) at low energy as needed for low temperature umklapp processes; however, our results suggest that such states are intrinsically present in the hypothetical guest-free tin clathrates, like calculated by Myles et al. ${ }^{44}$ Therefore, the relative effect of guest atoms will be small compared to silicon and germanium clathrates where such low energy states are not present in the empty clathrate. The similar thermal conductivities for $\mathrm{Rb}_{8} \mathrm{Sn}_{44} \square 2$ and $\mathrm{Cs}_{8} \mathrm{Sn}_{44} \square 2$ are attributed to these intrinsic low energy phonon states, which reduce the effect of guest atom substitution. To achieve interaction between rattling modes and all acoustic branches, the guest atom must, as demonstrated, not only be heavier, but also have smaller volume. Alternatively the cages confining the guest atoms must be even bigger. These consideration are contained within the expression: $\hbar \omega_{\text {Guest }} \propto \sqrt{V_{\text {guest }} /\left(m_{\text {guest }} \cdot V_{\text {cage }}\right)}$, which was used to approximate the ratio of rattling energies. In the case of $\mathrm{A}_{8} \mathrm{Ga}_{16} \mathrm{Ge}_{30}, \mathrm{~A}=\mathrm{Eu}, \mathrm{Ba}, \mathrm{Sr}$ compared by Bentien et al. ${ }^{17}$ the thermal conductivity of $\mathrm{Eu}_{8} \mathrm{Ga}_{16} \mathrm{Ge}_{30}$ is the lowest of the three compounds. This is in accordance with the Eu guest atom being both smaller and heavier than $\mathrm{Ba}$ and $\mathrm{Sr}$.

\section{CONCLUSION}

A low energy phonon mode at $\approx 3.5 \mathrm{meV}$ is observed for $\mathrm{Rb}_{8} \mathrm{Sn}_{44} \square 2$ and the temperature dependence indicates that it has significant spectral weight Sn host atoms in agreement with theoretical calculations. The lowest energy mode in the tin clathrates is, therefore, not related to the rattling of the guest atoms, but due to the intrinsically low energies of the host structure phonon branches. The guest mode does not hybridize with transverse acoustic modes, thus the guest atom effect is reduced with regard to lowering the thermal conductivity.

The guest rattling energies of $\mathrm{Rb}_{8} \mathrm{Sn}_{44} \square_{2}$ and $\mathrm{Cs}_{8} \mathrm{Sn}_{44} \square 2$ are by both experiment and theory found to be almost identical despite the large mass difference: $\hbar \omega_{C s} /\left.\hbar \omega_{R b}\right|_{E x p}$. $=0.98(1)$ and $\hbar \omega_{C S} /\left.\hbar \omega_{R b}\right|_{\text {Calc. }}=1.0$. To achieve maximal effect on thermal conductivity, heavy guest atoms must be combined with large cages. The similar room temperature thermal conductivity of $\mathrm{Rb}_{8} \mathrm{Sn}_{44} \square_{2}$ and $\mathrm{Cs}_{8} \mathrm{Sn}_{44} \square_{2}$ is explained by the similar rattler energies and lack of interaction with transverse acoustic phonons.

\section{ACKNOWLEDGMENTS}

The authors are thankful for financial support from Danish National Research Foundation (Center for Materials Crystallography), and the Danish Research Council for Nature and Universe (Danscatt). The authors are appreciating the beamtime allocated for the experiment at FRM-II in Munich, Germany.

${ }^{1}$ A. J. Minnich, M. S. Dresselhaus, Z. F. Ren, and G. Chen, Energy Environ. Sci. 2, 466 (2009).

${ }^{2}$ K. F. Hsu, S. Loo, F. Guo, W. Chen, J. S. Dyck, C. Uher, T. Hogan, E. K. Polychroniadis, and M. G. Kanatzidis, Science 303, 818 (2004).

${ }^{3}$ Z. H. Dughaish, Physica B 322, 205 (2002).

${ }^{4}$ G. S. Nolas, J. L. Cohn, G. A. Slack, and S. B. Schujman, Appl. Phys. Lett. 73, 178 (1998).

${ }^{5}$ G. S. Nolas, B. C. Chakoumakos, B. Mahieu, G. J. Long, and T. J. R. Weakley, Chem. Mater. 12, 1947 (2000).

${ }^{6}$ R. P. Hermann, W. Schweika, O. Leupold, R. Ruffer, G. S. Nolas, F. Grandjean, and G. J. Long, Phys. Rev. B 72, 174301 (2005).

${ }^{7}$ M. Christensen, S. Johnsen, F. Juranyi, and B. B. Iversen, J. Appl. Phys. 105, 073508 (2009).

${ }^{8}$ M. Christensen, S. Johnsen, and B. B. Iversen, Dalton Trans. 39, 978 (2010).

${ }^{9}$ N. P. Blake, D. Bryan, S. Latturner, L. Mollnitz, G. D. Stucky, and H. Metiu, J. Chem. Phys. 114, 10063 (2001).

${ }^{10}$ In literature the term, "rattler" has been loosely defined and some ambiguity surrounds its use (Refs. 4, 11, and 40). In the present paper we use the term "rattler" for guest atoms exhibiting excessive thermal motion vibrating at low energy.

${ }^{11}$ M. Christensen, A. B. Abrahamsen, N. B. Christensen, F. Juranyi, N. H. Andersen, K. Lefmann, J. Andreasson, C. R. H. Bahl, and B. B. Iversen, Nature Mater. 7, 811 (2008).

${ }^{12}$ J. M. Ziman, Electrons and Phonons: The Theory of Transport Phenomena in Solids (Clarendon, Oxford, 1960).

${ }^{13}$ E. S. Toberer, A. Zevalkink, and G. J. Snyder, J. Mater. Chem. 21, 15843 (2011).

${ }^{14}$ M. C. Karamarg, P. G. Klemens, F. P. Lipschul, and C. A. Reynolds, Phys. Rev. B 5, 2856 (1972).

${ }^{15}$ C. J. Glassbrenner and G. A. Slack, Phys. Rev. 134, 1058 (1964).

${ }^{16}$ B. C. Sales, B. C. Chakoumakos, R. Jin, J. R. Thompson, and D. Mandrus, Phys. Rev. B 63, 245113 (2001).

${ }^{17}$ A. Bentien, M. Christensen, J. D. Bryan, A. Sanchez, S. Paschen, F. Steglich, G. D. Stucky, and B. B. Iversen, Phys. Rev. B 69, 045107 (2004).

${ }^{18}$ L. Y. Qiu, I. P. Swainson, G. S. Nolas, and M. A. White, Phys. Rev. B 70, 035208 (2004).

${ }^{19}$ D. Nataraj, J. Nagao, M. Ferhat, and T. Ebinuma, J. Appl. Phys. 93, 2424 (2003).

${ }^{20}$ J. L. Cohn, G. S. Nolas, V. Fessatidis, T. H. Metcalf, and G. A. Slack, Phys. Rev. Lett. 82, 779 (1999).

${ }^{21}$ A. Kaltzoglou, T. Fassler, M. Christensen, S. Johnsen, B. Iversen, I. Presniakov, A. Sobolev, and A. Shevelkov, J. Mater. Chem. 18, 5630 (2008).

${ }^{22}$ F. Dubois and T. F. Fassler, J. Am. Chem. Soc. 127, 3264 (2005).

${ }^{23}$ T. Unruh, J. Neuhaus, and W. Petry, Nucl. Instrum. Methods Phys. Res. A 580, 1414 (2007).

${ }^{24} \mathrm{~J}$. Wuttke, see http://apps.jcns.fz-juelich.de/doku/frida/start, last accessed February 18, 2013.

${ }^{25}$ G. Kresse and J. Hafner, Phys. Rev. B 47, 558 (1993).

${ }^{26}$ G. Kresse and J. Furthmuller, Phys. Rev. B 54, 11169 (1996).

${ }^{27}$ J. P. Perdew, K. Burke, and M. Ernzerhof, Phys. Rev. Lett. 77, 3865 (1996).

${ }^{28}$ P. E. Blochl, Phys. Rev. B 50, 17953 (1994).

${ }^{29}$ G. Kresse and D. Joubert, Phys. Rev. B 59, 1758 (1999).

${ }^{30}$ V. S. Oskotskii, Sov. Phys. Solid State 9, 420 (1967).

${ }^{31}$ M. M. Koza, M. R. Johnson, H. Mutka, M. Rotter, N. Nasir, A. Grytsiv, and P. Rogl, Phys. Rev. B 82, 214301 (2010).

${ }^{32}$ M. Christensen, F. Juranyi, and B. B. Iversen, Physica B 385, 505 (2006).

${ }^{33}$ J. J. Dong, O. F. Sankey, and C. W. Myles, Phys. Rev. Lett. 86, 2361 (2001).

${ }^{34}$ J. S. Tse, Z. Li, and K. Uehara, Europhys. Lett. 56, 261 (2001).

${ }^{35}$ H. Shimizu, T. Imai, T. Kume, S. Sasaki, A. Kaltzoglou, and T. F. Fassler, Chem. Phys. Lett. 464, 54 (2008).

${ }^{36}$ P. Rogl, N. Melnychenko-Koblyuk, A. Grytsiv, L. Fornasari, H. Kaldarar, H. Michor, F. Rohrbacher, M. Koza, E. Royanian, E. Bauer, M. Rotter, H. Schmid, F. Marabelli, A. Devishvili, M. Doerr, and G. Giester, J. Phys.: Condens. Matter 19, 216223 (2007).

${ }^{37}$ B. Fultz, Prog. Mater. Sci. 55, 247 (2010). 
${ }^{38}$ K. Iwasa, M. Kohgi, H. Sugawara, and H. Sato, Physica B 378-380, 194 (2006).

${ }^{39}$ H. Schober, H. Itoh, A. Klapproth, V. Chihaia, and W. F. Kuhs, Eur. Phys. J. E 12, 41 (2003).

${ }^{40}$ M. M. Koza, M. R. Johnson, R. Viennois, H. Mutka, L. Girard, and D. Ravot, Nature Mater. 7, 805 (2008).
${ }^{41}$ L. Pauling, J. Am. Chem. Soc. 49, 765 (1927).

${ }^{42}$ C. W. Myles, K. Biswas, and E. Nenghabi, Physica B 401, 695 (2007).

${ }^{43}$ C. P. Yang, H. Wang, K. Iwasa, M. Kohgi, H. Sugawara, and H. Sato, J. Phys.: Condens. Matter 19, 226214 (2007).

${ }^{44}$ C. W. Myles, J. Dong, O. F. Sankey, C. A. Kendziora, and G. S. Nolas, Phys. Rev. B 65, 235208 (2002). 